\title{
Mixed problems for degenerate abstract parabolic equations and applications
}

\section{VELI. B. SHAKHMUROV ${ }^{1,2}$ and AIDA SAHMUROVA ${ }^{3}$}

\section{ABSTRACT.}

Degenerate abstract parabolic equations with variable coefficients are studied. Here the boundary conditions are nonlocal. The maximal regularity properties of solutions for elliptic and parabolic problems and Strichartz type estimates in mixed Lebesgue spaces are obtained. Moreover, the existence and uniqueness of optimal regular solution of mixed problem for nonlinear parabolic equation is established. Note that, these problems arise in fluid mechanics and environmental engineering.

\section{REFERENCES}

[1] Amann, H., Linear and quasilinear parabolic problems, 1, 2, Birkhauser, Basel, 1995

[2] Agarwal, R., O'Regan, D., and Shakhmurov, V. B., Separable anisotropic differential operators in weighted abstract spaces and applications, J. Math. Anal. Appl., 338 (2008), 970-983

[3] Ashyralyev, A., Claudio, C. and Piskarev, S., On well-posedness of difference schemes for abstract elliptic problems in $L_{p}$ spaces, Numer. Func. Anal. Optim., 29 (2008), 43-65

[4] Favini, A. and Yagi, A., Degenerate Differential Equations in Banach Spaces, Taylor \& Francis, Dekker, NewYork, 1999

[5] Shakhmurov, V., Embedding theorems and maximal regular differential operator equations in Banach-valued function spaces, J. Inequal. Appl., (4) (2005), 605-620

[6] Shakhmurov, V. B., Linear and nonlinear abstract equations with parameters, Nonlinear Anal., 73 (2010), 2383-2397

[7] Shakhmurov, V. B., Regular degenerate separable differential operators and applications, Potential Anal., 35 (2011), No. 3, 201-212

[8] Shakhmurov, V. B., Coercive boundary value problems for regular degenerate differential-operator equations, J. Math. Anal. Appl., 292 (2004), No. 2, 605-620

[9] Shakhmurov, V. B., and Shahmurova, A., Nonlinear abstract boundary value problems atmospheric dispersion of pollutants, Nonlinear Anal. Real World Appl., 11 (2010), No. 2, 932-951

[10] Weis, L., Operator-valued Fourier multiplier theorems and maximal $L_{p}$ regularity, Math. Ann., 319 (2001), 735-758

[11] Yakubov, S. and Yakubov, Y., Differential-operator Equations. Ordinary and Partial Differential Equations, Chapman and Hall /CRC, Boca Raton, 2000

${ }^{1}$ OKAN UNIVERSITY

MECHANICAL ENGINEERING

AKFIRAT, TUZLA 34959 ISTANBUL, TURKEY

${ }^{2}$ KHAZAR UNIVERSITY

MATHEMATICS

BAKU, AZERBAIJAN

E-mail address: veli.sahmurov@okan.edu.tr

Received: 05.09.2016; In revised form: 06.07.2018; Accepted: 13.07.2018

2010 Mathematics Subject Classification. 35A01 35J56 35K51 47G40.

Key words and phrases. differential-operator equations, degenerate PDE, semigroups of operators, nonlinear problems, separable differential operators, positive operators in Banach spaces.

Corresponding author: Veli. B. Shakhmurov; veli.sahmurov@okan.edu.tr 
${ }^{3}$ OKAN UNIVERSITY

ENVIRONMENTAL ENGINEERING

AKFIRAT, TUZLA 34959 ISTANBUL, TURKEY

E-mail address: aida.sahmurovadokan.edu.tr 